\title{
Existence and blow up of solutions to a Petrovsky equation with memory and nonlinear source term
}

Faramarz Tahamtani* and Mohammad Shahrouzi

* Correspondence: tahamtani@shirazu.ac.ir Department of Mathematics, College of Sciences, Shiraz University, Shiraz, 71454, Iran

\section{Abstract}

We consider the semilinear Petrovsky equation

$$
u_{t t}+\Delta^{2} u-\int_{0}^{t} g(t-s) \Delta^{2} u(s) d s=|u|^{p} u
$$

in a bounded domain and prove the existence of weak solutions. Furthermore, we show that there are solutions under some conditions on initial data which blow up in finite time with non-positive initial energy as well as positive initial energy.

Estimates of the lifespan of solutions are also given.

Mathematics Subject Classification (2000): 35L35; 35L75; 37B25.

Keywords: viscoelasticity, existence, blow-up, life-span, negative initial energy, positive initial energy

\section{Introduction}

In this article, we concerned with the problem

$$
\begin{gathered}
u_{t t}+\Delta^{2} u-\int_{0}^{t} g(t-s) \Delta^{2} u(s) d s=|u|^{p} u, \quad x \in \Omega, \tau>0 \\
u(x, t)=\partial_{\nu} u(x, t)=0, \quad x \in \partial \Omega, t \geq 0 \\
u(x, 0)=u_{0}(x), \quad u_{t}(x, 0)=u_{1}(x), \quad x \in \Omega
\end{gathered}
$$

where $\Omega \subset R^{n}$ is a bounded domain with smooth boundary $\partial \Omega$ in order that the divergence theorem can be applied. $v$ is the unit normal vector pointing toward the exterior of $\Omega$ and $p>0$. Here, $g$ represents the kernel of the memory term satisfying some conditions to be specified later.

In the absence of the viscoelastic term, i.e., $(g=0)$, we motivate our article by presenting some results related to initial-boundary value Petrovsky problem

$$
\begin{gathered}
u_{t t}+\Delta^{2}{ }_{u}=f\left(u, u_{t}\right), \quad x \in \Omega, \quad t>0 \\
u(x, t)=\partial_{\nu} u(x, t)=0, \quad x \in \partial \Omega, t \geq 0 \\
u(x, 0)=u_{0}(x), \quad u_{t}(x, 0)=u_{1}(x), \quad x \in \Omega .
\end{gathered}
$$

(c) 2012 Tahamtani and Shahrouzi; licensee Springer. This is an Open Access article distributed under the terms of the Creative Commons Attribution License (http://creativecommons.org/licenses/by/2.0), which permits unrestricted use, distribution, and reproduction in any medium, provided the original work is properly cited. 
Research of global existence, blow-up and energy decay of solutions for the initial boundary value problem (1.2) has attracted a lot of articles (see [1-4] and references there in).

Amroun and Benaissa [1] investigated (1.2) with $f\left(u, u_{t}\right)=b|u|^{p-2} u-h\left(u_{t}\right)$ and proved the global existence of solutions by means of the stable set method in $H_{0}^{2}(\Omega)$ combined with the Faedo-Galerkin procedure. In [3], Messaoudi studied problem (1.2) with $f\left(u, u_{t}\right)=b|u|^{p-2} u-a\left|u_{t}\right|^{m-2} u_{t}$. He proved the existence of a local weak solution and showed that this solution blows up in finite time with negative initial energy if $p>m$.

In the presence of the viscoelastic terms, Rivera et al. [5] considered the plate model:

$$
u_{t t}+\Delta^{2} u-\int_{0}^{t} g(t-s) \Delta^{2} u(s) d s=0
$$

in a bounded domain $\Omega \subset R^{N}$ and showed that the energy of solution decay exponentially provided the kernel function also decay exponentially. For more related results about the existence, finite time blow-up and asymptotic properties, we refer the reader to [5-16].

In the present article, we devote our study to problem (1.1). We will prove the existence of weak solutions under some appropriate assumptions on the function $g$ and blow-up behavior of solutions. In order to obtain the existence of solutions, we use the Faedo-Galerkin method and to get the blow-up properties of solutions with non-positive and positive initial energy, we modify the method in [17]. Estimates for the blowup time $T^{*}$ are also given.

\section{Preliminaries}

We define the energy function related with problem (1.1) is given by

$$
E(t)=\frac{1}{2}\left[\left\|u_{t}\right\|^{2}+\left(1-\int_{0}^{t} g(s) d s\right)\|\Delta u\|^{2}+(g \odot \Delta u)(t)\right]-\frac{1}{p+2}\|u\|_{p+2}^{p+2},
$$

where

$$
(g \odot v)(t)=\int_{0}^{t} g(t-s)\|v(t)-v(s)\|_{2}^{2} d s .
$$

We denote by $\|\cdot\|_{k}$, the $L^{k}$-norm over $\Omega$. In particular, the $L^{2}$-norm is denoted $\|\cdot\|_{2}$. We use the familiar function spaces $H_{0}^{2}, H^{4}$ and throughout this article we assume $u_{0} \in H_{0}^{2}(\Omega) \cap H^{4}(\Omega)$ and $u_{1} \in H_{0}^{2}(\Omega) \cap L^{2}(\Omega)$.

In the sequel, we state some hypotheses and three well-known lemmas that will be needed later.

(A1) $p$ satisfies

$$
\begin{gathered}
0<p \leq \infty \quad(N \leq 4), \\
0<p \leq \frac{2(N-2)}{N-4} \quad(N \geq 5) .
\end{gathered}
$$


(A2) $g$ is a positive bounded $C^{1}$ function satisfying $g(0)>0$, and for all $t>0$

$$
1-\int_{0}^{\infty} g(t) d s=l>0,
$$

also there exists positive constants $L_{0}, L_{1}$ such that

$$
-L_{0} \leq g^{\prime}(t) \leq 0, \quad 0 \leq g^{\prime \prime}(t) \leq L_{1} .
$$

Lemma 1 (Sobolev-Poincare's inequality). Let $p$ be a number that satisfies (A1), then there is a constant $C_{*}=C(\Omega, p)$ such that

$$
\|u\|_{p} \leq C_{*}\|\Delta u\|_{2}, \quad u \in H_{0}^{2}(\Omega)
$$

Lemma 2 [4]. Let $\delta>0$ and $B(t) \in C^{2}(0, \infty)$ be a nonnegative function satisfying

$$
B^{\prime \prime}(t)-4(\delta+1) B^{\prime}(t)+4(\delta+1) B(t) \geq 0 .
$$

If

$$
B^{\prime}(0)>r_{2} B(0)+K_{0}
$$

with $r_{2}=2(\delta+1)-2 \sqrt{\delta(\delta+1)}$, then $B^{\prime}(t)>K_{0}$ for $t>0$, where $K_{0}$ is a constant.

Lemma 3 [4]. If $Y(t)$ is a non-increasing function on $\left[t_{0}, \infty\right)$ and satisfies the differential inequality

$$
Y^{\prime}(t)^{2} \geq a+b Y(t)^{2+\delta^{-1}} \quad \text { for } \quad t \geq t_{0} \geq 0,
$$

where $a>0, \delta>0$ and $b \in R$, then there exists a finite time $T^{*}$ such that

$$
\lim _{t \rightarrow T^{*-}} Y(t)=0
$$

Upper bounds for $T^{*}$ is estimated as follows:

(i) If $b<0$, then

$$
T^{*} \leq t_{0}+\frac{1}{\sqrt{-b}} \ln \frac{\sqrt{\frac{-a}{b}}}{\sqrt{\frac{-a}{b}-Y\left(t_{0}\right)}}
$$

(ii) If $b=0$, then

$$
T^{*} \leq t_{0}+\frac{Y\left(t_{0}\right)}{Y^{\prime}\left(t_{0}\right)} .
$$

(iii) If $b>0$, then

$$
T^{*} \leq \frac{Y\left(t_{0}\right)}{\sqrt{a}},
$$


or

$$
T^{*} \leq t_{0}+2^{\frac{3 \delta+1}{2 \delta}} \frac{c \delta}{\sqrt{a}}\left\{1-\left[1+c Y\left(t_{0}\right)\right]^{\frac{-1}{2 \delta}}\right\}
$$

where $_{c}=\left(\frac{a}{b}\right)^{2+\frac{1}{\delta}}$.

\section{Existence of solutions}

In this section, we are going to obtain the existence of weak solutions to the problem (1.1) using Faedo-Galerkin's approximation.

Theorem 1 Let the assumptions (A1)-(A3) hold. Then there exists at least a solution $u$ of (1.1) satisfying

$$
\begin{aligned}
u \in L^{\infty}\left(0, \infty ; H_{0}^{2}(\Omega) \cap\right. & \left.H^{4}(\Omega)\right), \quad u^{\prime} \in L^{\infty}\left(0, \infty ; H_{0}^{2}(\Omega) \cap L^{2}(\Omega)\right), \\
& u^{\prime \prime} \in L^{\infty}\left(0, \infty ; L^{2}(\Omega)\right)
\end{aligned}
$$

and

$$
\begin{aligned}
& u(x, t) \rightarrow u_{0}(x) \quad \text { in } H_{0}^{2}(\Omega) \cap H^{4}(\Omega) \\
& u^{\prime}(x, t) \rightarrow u_{1}(x) \quad \text { in } H_{0}^{2}(\Omega) \cap L^{2}(\Omega)
\end{aligned}
$$

as $t \rightarrow 0$.

Proof We choose a basis $\left\{\omega_{k}\right\}(k=1,2, \ldots)$ in $H_{0}^{2}(\Omega) \cap H^{4}(\Omega)$ which is orthonormal in $L^{2}(\Omega)$ and $\omega_{k}$ being the eigenfunctions of biharmonic operator subject to the homogeneous Dirichlet boundary condition.

Let $V_{m}$ be the subspace of $H_{0}^{2}(\Omega) \cap H^{4}(\Omega)$ generated by the first $m$ vectors. Define

$$
u_{m}(t)=\sum_{k=1}^{m} d_{m}^{k}(t) \omega_{k}
$$

where $u_{m}(t)$ is the solution of the following Cauchy problem

$$
\begin{gathered}
\left(u_{m}^{\prime \prime}(t), \omega_{k}\right)+\left(\Delta u_{m}(t), \Delta \omega_{k}\right)-\int_{0}^{t}(t-s)\left(\Delta u_{m}(s), \Delta \omega_{k}\right) d s \\
-\left(\left|u_{m}(t)\right|^{p} u_{m}(t), \omega_{k}\right)=0 \quad \forall k=1, m .
\end{gathered}
$$

with the initial conditions (when $m \rightarrow \infty$ )

$$
\left\{\begin{array}{l}
u_{m}(0)=\sum_{k=1}^{m}\left(u_{m}(0), \omega_{k}\right) \omega_{k} \rightarrow u_{0} \text { in } H_{0}^{2}(\Omega) \cap H^{4}(\Omega) \\
u_{m}^{\prime}(0)=\sum_{k=1}^{m}\left(u_{m}^{\prime}(0), \omega_{k}\right) \omega_{k} \rightarrow u_{1} \text { in } H_{0}^{2}(\Omega) \cap L^{2}(\Omega)
\end{array}\right.
$$

The approximate systems (3.3) and (3.4) are the normal one of differential equations which has a solution in $\left[0, T_{m}\right)$ for some $T_{m}>0$. The solution can be extended to the $[0, T]$ for any given $T>0$ by the first estimate below.

First estimation. Substituting $u_{m}^{\prime}(t)$ instead of $\omega_{k}$ in (3.3), we find

$$
\frac{d}{d t}\left(\frac{1}{2}\left\|u_{m}^{\prime}\right\|^{2}+\frac{1}{2}\left\|\Delta u_{m}\right\|^{2}-\frac{\left\|u_{m}\right\|_{p+2}^{p+2}}{p+2}\right)-\int_{0}^{t} g(t-s)\left(\Delta u_{m}(s), \Delta u_{m}^{\prime}(t)\right) d s=0 .
$$


Simple calculation similar to [11] yield

$$
\begin{aligned}
& -\int_{0}^{t} g(t-s)\left(\Delta u_{m}(s), \Delta u_{m}^{\prime}(t)\right) d s=-\int_{0}^{t} g(t-s) \int_{\Omega} \Delta u_{m}(t) \Delta u_{m}^{\prime}(t) d x d s \\
& \quad-\int_{0}^{t} g(t-s) \int_{\Omega}\left(\Delta u_{m}(s)-\Delta u_{m}(t)\right) \Delta u_{m}^{\prime}(t) d x d s \\
& =\frac{1}{2} \int_{0}^{t} g(t-s) \frac{d}{d t}\left\|\Delta u_{m}(s)-\Delta u_{m}(t)\right\|^{2} d s-\frac{1}{2} \int_{0}^{t} g(t-s) \frac{d}{d t}\left\|\Delta u_{m}(t)\right\|^{2} d s \\
& =\frac{1}{2} \frac{d}{d t}\left(g \odot \Delta u_{m}\right)(t)-\frac{1}{2}\left(g^{\prime} \odot \Delta u_{m}\right)(t)-\frac{1}{2} \frac{d}{d t} \int_{0}^{t} g(s) d s\left\|\Delta u_{m}(t)\right\|^{2} d s \\
& +\frac{1}{2} g(t)\left\|\Delta u_{m}(t)\right\|^{2} .
\end{aligned}
$$

Combining (3.5) and (3.6), we find

$$
\begin{gathered}
\frac{d}{d t}\left(\frac{1}{2}\left\|u_{m}^{\prime}\right\|^{2}+\frac{1}{2}\left(1-\int_{0}^{t} g(s) d s\right)\left\|\Delta u_{m}\right\|^{2}+\frac{1}{2}\left(g \odot \Delta u_{m}\right)(t)-\frac{\left\|u_{m}\right\|_{p+2}^{p+2}}{p+2}\right) \\
=\frac{1}{2}\left(g^{\prime} \odot \Delta u_{m}\right)(t)-\frac{1}{2} g(t)\left\|\Delta u_{m}(t)\right\|^{2},
\end{gathered}
$$

integrating (3.7) over $(0, t)$ and using assumption (A3) we infer that

$$
\left\|u_{m}^{\prime}\right\|^{2}+\left\|\Delta u_{m}\right\|^{2}+\left(g \odot \Delta u_{m}\right)(t)-\left\|u_{m}\right\|_{p+2}^{p+2} \leq C_{1}
$$

where $C_{1}$ is a positive constant depending only on $\left\|u_{0}\right\|,\left\|u_{1}\right\|, p$, and $l$. It follows from (3.8) that

$$
\left\{\begin{array}{c}
\left\{u_{m}\right\} \text { is uniformly bounded in } L^{\infty}\left(0, T ; H_{0}^{2}(\Omega)\right) \\
\left\{u_{m}^{\prime}\right\} \text { is uniformly bounded in } L^{\infty}\left(0, T ; L^{2}(\Omega)\right)
\end{array}\right.
$$

Second estimation. Differentiating (3.3) with respect to $t$, we get

$$
\begin{gathered}
\left(u_{m}^{\prime \prime \prime}(t), \omega_{k}\right)+\left(\Delta u_{m}^{\prime}(t), \Delta \omega_{k}\right)-\int_{0}^{t} g^{\prime}(t-s)\left(\Delta u_{m}(s), \Delta \omega_{k}\right) d s \\
-g(0)\left(\Delta u_{m}(t), \Delta \omega_{k}\right)-(p+1)\left(\left|u_{m}(t)\right|^{p} u_{m}^{\prime}(t), \omega_{k}\right)=0 .
\end{gathered}
$$

If we substitute $u_{m}^{\prime \prime}(t)$ instead of $\omega_{k}$ in (3.10), it holds that

$$
\begin{gathered}
\frac{d}{d t}\left(\frac{1}{2}\left\|u_{m}^{\prime \prime}\right\|^{2}+\frac{1}{2}\left\|\Delta u_{m}^{\prime}\right\|^{2}\right)-\frac{d}{d t} \int_{0}^{t} g^{\prime}(t-s)\left(\Delta u_{m}(s), \Delta u_{m}^{\prime}(t)\right) d s \\
+\int_{0}^{t} g^{\prime \prime}(t-s)\left(\Delta u_{m}(s), \Delta u_{m}^{\prime}(t)\right) d s+g^{\prime}(0)\left(\Delta u_{m}(t), \Delta u_{m}^{\prime}(t)\right) \\
-g(0) \frac{d}{d t}\left(\Delta u_{m}(t), \Delta u_{m}^{\prime}(t)\right)+g(0)\left(\Delta u_{m}^{\prime}(t), \Delta u_{m}^{\prime}(t)\right) \\
-(p+1)\left(\left|u_{m}(t)\right|^{p} u_{m}^{\prime}(t), u_{m}^{\prime \prime}(t)\right)=0 .
\end{gathered}
$$


Since $H^{2}(\Omega) \otimes L^{2 p+2}(\Omega)$, using Lemma 2, Hölder and Young's inequalities and (3.8)

$$
\begin{aligned}
&\left|(p+1)\left(\left|u_{m}(t)\right|^{p} u_{m}^{\prime}(t), u_{m}^{\prime \prime}(t)\right)\right| \leq(p+1)\left\|u_{m}(t)\right\|_{2 p+2}^{p} \cdot\left\|u_{m}^{\prime}(t)\right\|_{2 p+2} \cdot\left\|u_{m}^{\prime \prime}(t)\right\|_{2} \\
& \leq C(\gamma)\left\|\Delta u_{m}^{\prime}(t)\right\|^{2}+\gamma\left\|u_{m}^{\prime \prime}(t)\right\|^{2} .
\end{aligned}
$$

Combining the relations (3.11), (3.12) and integrating over $(0, t)$ for all $t \in[0, T]$ with arbitrary fixed $T$, we obtain

$$
\begin{gathered}
\frac{1}{2}\left\|u_{m}^{\prime \prime}\right\|^{2}+\frac{1}{2}\left\|\Delta u_{m}^{\prime}\right\|^{2} \leq \frac{1}{2}\left\|u_{m}^{\prime \prime}(0)\right\|^{2}+\int_{0}^{t} g^{\prime}(t-s)\left(\Delta u_{m}(s), \Delta u_{m}^{\prime}(t)\right) d s \\
+\frac{1}{2}\left\|\Delta u_{m}^{\prime}(0)\right\|^{2}-\int_{0}^{t} \int_{0}^{\tau} g^{\prime \prime}(\tau-s)\left(\Delta u_{m}(s), \Delta u_{m}^{\prime}(\tau)\right) d s d \tau \\
-g^{\prime}(0) \int_{0}^{t}\left(\Delta u_{m}(s), \Delta u_{m}^{\prime}(s)\right)+g(0)\left(\Delta u_{m}(t), \Delta u_{m}^{\prime}(t)\right) \\
-g(0)\left(\Delta u_{m}(0), \Delta u_{m}^{\prime}(0)\right)-g(0) \int_{0}^{t}\left\|\Delta u_{m}^{\prime}(s)\right\|^{2} d s \\
+C(\gamma) \int_{0}^{t}\left\|\Delta u_{m}^{\prime}(s)\right\|^{2} d s+\gamma \int_{0}^{t}\left\|u_{m}^{\prime \prime}(s)\right\|^{2} d s .
\end{gathered}
$$

From (3.4) and (3.8), we deduce that

$$
\left|\frac{1}{2}\left\|\Delta u_{m}^{\prime}(0)\right\|^{2}-g(0)\left(\Delta u_{m}(0), \Delta u_{m}^{\prime}(0)\right)\right| \leq L_{2},
$$

where $L_{2}$ is a positive constant independent of $m$. In the following, we find the upper bound for $\left\|u_{m}^{\prime \prime}(0)\right\|^{2}$. Again we substitute $u_{m}^{\prime \prime}(t)$ instead of $\omega_{k}$ in (3.3), and choosing $t=$ 0 , we arrive at

$$
\left(u_{m}^{\prime \prime}(0), u_{m}^{\prime \prime}(0)\right)+\left(\Delta u_{m}(0), \Delta u_{m}^{\prime \prime}(0)\right)-\left(\left|u_{m}(0)\right|^{p} u_{m}(0), u_{m}^{\prime \prime}(0)\right)=0,
$$

which combined with the Green's formula imply

$$
\left\|u_{m}^{\prime \prime}(0)\right\|^{2}+\left(\Delta^{2} u_{m}(0), u_{m}^{\prime \prime}(0)\right)-\left(\left|u_{m}(0)\right|^{p} u_{m}(0), u_{m}^{\prime \prime}(0)\right)=0 .
$$

By using (A1), (3.4) and Young's inequality, we deduce that

$$
\left\|u_{m}^{\prime \prime}(0)\right\| \leq L_{3},
$$

where $L_{3}>0$ is a constant independent of $m$. 
Owing to (3.8), (3.5) and Young's inequality with (A3), we deduce that

$$
\begin{aligned}
& \left.\mid \int_{0}^{t} g^{\prime}(t-s)\left(\Delta u_{m}(s), \Delta u_{m}^{\prime}(t)\right) d s\right)|=|\left(\Delta u_{m}^{\prime}(t), \int_{0}^{t} g^{\prime}(t-s) \Delta u_{m}(s) d s\right) \mid \\
& \leq \gamma\left\|\Delta u_{m}^{\prime}(t)\right\|^{2}+\frac{1}{4 \gamma} \int_{\Omega}\left(\int_{0}^{t} g^{\prime}(t-s) \Delta u_{m}(s) d s\right)^{2} d x \\
& \leq \gamma\left\|\Delta u_{m}^{\prime}(t)\right\|^{2}+\frac{L_{0}^{2}}{4 \gamma} \int_{0}^{t}\left\|\Delta u_{m}(s)\right\|^{2} d s \\
& \leq \gamma\left\|\Delta u_{m}^{\prime}(t)\right\|^{2}+L_{4}(T), \\
& \left|-\int_{0}^{t} \int_{0}^{\tau} g^{\prime \prime}(\tau-s)\left(\Delta u_{m}(s), \Delta u_{m}^{\prime}(\tau)\right) d s d \tau\right| \\
& =\int_{0}^{t}\left(\Delta u_{m}^{\prime}(\tau), \int_{0}^{\tau} g^{\prime \prime}(\tau-s) \Delta u_{m}(s) d s\right) d \tau \\
& \leq \frac{1}{2} \int_{0}^{t}\left\|\Delta u_{m}^{\prime}(s)\right\|^{2} d s+\frac{1}{2} \int_{0}^{t} \int_{\Omega}\left(\int_{0}^{\tau} g^{\prime \prime}(\tau-s) \Delta u_{m}(s) d s\right)^{2} d x d \tau \\
& \leq \frac{1}{2} \int_{0}^{t}\left\|\Delta u_{m}^{\prime}(s)\right\|^{2} d s+\frac{T L_{1}^{2}}{2} \int_{0}^{t}\left\|\Delta u_{m}(s)\right\|^{2} d s \\
& \leq \frac{1}{2} \int_{0}^{t}\left\|\Delta u_{m}^{\prime}(s)\right\|^{2} d s+L_{5}(T), \\
& \left|-g^{\prime}(0) \int_{0}^{t}\left(\Delta u_{m}(s), \Delta u_{m}^{\prime}(s)\right) d s\right| \leq L_{0} \int_{0}^{t}\left\|\Delta u_{m}^{\prime}(s)\right\|^{2} d s+L_{6}(T),
\end{aligned}
$$

and

$$
\left|g(0)\left(\Delta u_{m}(t), \Delta u_{m}^{\prime}(t)\right)\right| \leq \gamma\left\|\Delta u_{m}^{\prime}(t)\right\|^{2}+L_{7}(\gamma) .
$$

Now we choose $\gamma>0$ small enough and combining (A3), (3.8), (3.13), (3.14), and (3.16)-(3.20), we get

$$
\frac{1}{2}\left\|u_{m}^{\prime \prime}\right\|^{2}+\frac{1}{2}\left\|\Delta u_{m}^{\prime}\right\|^{2} \leq L_{8}\left(\int_{0}^{t}\left\|u_{m}^{\prime \prime}(s)\right\|^{2} d s+\int_{0}^{t}\left\|\Delta u_{m}^{\prime}(s)\right\|^{2} d s\right)+L_{9} .
$$

By using Gronwall's lemma we arrive at

$$
\frac{1}{2}\left\|u_{m}^{\prime \prime}\right\|^{2}+\frac{1}{2}\left\|\Delta u_{m}^{\prime}\right\|^{2} \leq L_{10}
$$

for all $t \in[0, T]$, and $L_{10}$ is a positive constant independent of $m$. Estimate (3.22) implies

$\left\{\left\{u_{m}^{\prime \prime}\right\}\right.$ is uniformly bounded in $L^{\infty}\left(0, T ; L^{2}(\Omega)\right)$

$\left\{\left\{u_{m}^{\prime}\right\}\right.$ is uniformly bounded in $L^{\infty}\left(0, T ; H_{0}^{2}(\Omega)\right)$ 
By attention to (3.9) and (3.23), there exists a subsequence $\left\{u_{i}\right\}$ of $\left\{u_{m}\right\}$ and a function $u$ such that

$$
\left\{\begin{array}{l}
u_{i} \rightarrow u \text { weakly star in } L^{\infty}\left(0, T ; H_{0}^{2}(\Omega)\right) \\
u_{i}^{\prime} \rightarrow u^{\prime} \text { weakly star in } L^{\infty}\left(0, T ; H_{0}^{2}(\Omega)\right) \\
u_{i}^{\prime \prime} \rightarrow u^{\prime \prime} \text { weakly star in } L^{\infty}\left(0, T ; L^{2}(\Omega)\right)
\end{array}\right.
$$

By Aubin-Lions compactness lemma [18], it follows from (3.24) that

$$
\left\{\begin{array}{l}
u_{i} \rightarrow u \text { strongly in } C\left([0, T] ; H_{0}^{2}(\Omega)\right) \\
\left.u_{i}^{\prime} \rightarrow u \text { strongly in } C([0, T]) ; L^{2}(\Omega)\right)
\end{array}\right.
$$

In the sequel we will deal with the nonlinear term. By (3.9) and Sobolev embedding theorem, we obtain

$$
\left\{\left|u_{m}\right|^{p} u_{m}\right\} \text { is uniformly bounded in } L^{\infty}\left(0, T ; L^{2}(\Omega)\right),
$$

and therefore we can extract a subsequence $\left\{u_{i}\right\}$ of $\left\{u_{m}\right\}$ such that

$$
\left|u_{i}\right|^{p} u_{i} \rightarrow|u|^{p} u \text { weakly star in } L^{\infty}\left(0, T ; L^{2}(\Omega)\right) .
$$

Applying (3.24), (3.27) and letting $i \rightarrow \infty$ in (3.3), we see that $u$ satisfies the equation. For the initial conditions by using (3.4), (3.25) and the simple inequality

$$
\left\|u-u_{0}\right\|_{H_{0}^{2}(\Omega)} \leq\left\|u-u_{i}\right\|_{H_{0}^{2}(\Omega)}+\left\|u_{i}-u_{i}(0)\right\|_{H_{0}^{2}(\Omega)}+\left\|u_{i}(0)-u_{0}\right\|_{H_{0}^{2}(\Omega)^{\prime}}
$$

we get the first initial condition immediately. In the similar way, we can show the second initial condition and the proof is complete.

\section{Blow-up of solutions}

In this section, we study blow-up property of solutions with non-positive initial energy as well as positive initial energy, and estimate the lifespan of solutions. For this purpose, we assume that $g$ is positive and $C^{1}$ function satisfying

$(A 4)$

$$
g(0)>0, \quad g^{\prime}(s) \leq 0, \quad 1-\int_{0}^{\infty} g(s) d s=l>0,
$$

and we make the following extra assumption on $g$

(A5)

$$
\int_{0}^{\infty} g(s) d s<\frac{p}{1+p}
$$

From (2.1), (A4) and Lemma 1, we have

$$
\begin{aligned}
E(t) \geq & \frac{1}{2}\left[\left(1-\int_{0}^{t} g(s) d s\right)\|\Delta u\|^{2}+(g \odot \Delta u)(t)\right]-\frac{1}{p+2}\|u\|_{p+2}^{p+2} \\
& \left.\geq \frac{1}{2}\left[l\|\Delta u\|^{2}+g \odot \Delta u\right)(t)\right]-\frac{C_{1}^{p+2} l^{\frac{p+2}{2}}}{p+2}\|\Delta u\|^{p+2} \\
& \geq G\left(\sqrt{l\|\Delta u\|^{2}+(g \odot \Delta u)(t)}\right), \quad t \geq 0,
\end{aligned}
$$


where $G(\lambda)=\frac{1}{2} \lambda^{2}-\frac{C_{1}^{p+2}}{p+2} \lambda^{p+2}, \quad C_{1}=\frac{C_{*}}{\sqrt{l}}$. It is easy to verify that $G(\lambda)$ has a maximum at $\lambda_{1}=C_{1}^{-\frac{p+2}{p}}$ and the maximum value is $E_{1}=\frac{p}{2 p+4} C_{1}^{-\frac{2 p+4}{p}}$.

Lemma 4 Let (A4) hold andu be a local solution of (1.1). Then $E(t)$ is a non-increasing function on $[0, T]$ and

$$
\frac{d}{d t} E(t)=\frac{1}{2}\left(g^{\prime} \odot \Delta u\right)(t)-\frac{1}{2} g(t)\|\Delta u\|^{2} \leq 0,
$$

for almost every $t \in[0, T]$.

Proof Multiplying (1.1) by $u_{t}$, integrating over $\Omega$, and finally integrating by parts, we obtain (4.2) for any regular solution. Then by density arguments, we have the result.

Lemma 5 Let (A4) hold and $u$ be a local solution of (1.1) with initial data satisfying $E(0)<E_{1}$ and $l^{\frac{1}{2}}\left\|\Delta u_{0}\right\|>\lambda_{1}$. Then there exists $\lambda_{2}>\lambda_{1}$ such that

$$
l\|\Delta u\|^{2}+(g \odot \Delta u)(t) \geq \lambda_{2}^{2} .
$$

Proof See Li and Tsai [11].

The choice of the functional is standard (see [19])

$$
\psi(t)=\|u\|^{2} .
$$

It is clear that

$$
\psi^{\prime}(t)=2\left(u, u_{t}\right),
$$

and from (1.1)

$$
\psi^{\prime \prime}(t)=2\left\|u_{t}\right\|^{2}-2\|\Delta u\|^{2}+2\|u\|_{p+2}^{p+2}+2 \int_{0}^{t} g(t-s)(\Delta u(t), \Delta u(s)) d s .
$$

Lemma 6 Let $u$ be a solution of (1.1) and (A4), (A5) hold, then we have

$$
\psi^{\prime \prime}(t)-(4+p) \int_{\Omega} u_{t}^{2} d x \geq m\left(l\|\Delta u\|^{2}+(g \odot \Delta u)(t)\right)-(4+2 p) E(0)
$$

where $m=(1+p)-\frac{1}{l}>0$.

Proof Using the Hölder and Young's inequalities, we arrive at

$$
\begin{aligned}
\int_{0}^{t} g(t-s)(\Delta u(t), \Delta u(s)) d s & \geq-\left[\frac{1}{2}(g \odot \Delta u)(t)+\frac{1}{2} \int_{0}^{t} g(s) d s\|\Delta u\|^{2}\right] \\
& +\int_{0}^{t} g(s) d s\|\Delta u\|^{2},
\end{aligned}
$$


therefore (4.6) becomes

$$
\begin{aligned}
\psi^{\prime \prime}(t)-(4+p)\left\|u_{t}\right\|^{2} & \geq-(2+p)\left\|u_{t}\right\|^{2}-2\|\Delta u\|^{2}-(g \odot \Delta u)(t) \\
& +\int_{0}^{t} g(s) d s\|\Delta u\|^{2}+2\|u\|_{p+2}^{p+2} .
\end{aligned}
$$

Then, using (4.2), we obtain

$$
\begin{array}{r}
\psi^{\prime \prime}(t)-(4+p)\left\|u_{t}\right\|^{2} \geq-(4+2 p) E(0)+p\|\Delta u\|^{2}+(1+p)(g \odot \Delta u)(t) \\
-(1+p) \int_{0}^{t} g(s) d s\|\Delta u\|^{2}-(2+p) \int_{0}^{t}\left(g^{\prime} \odot \Delta u\right)(s) d s
\end{array}
$$

and so by (2.5) and (A5), we deduce

$$
\begin{aligned}
\psi^{\prime \prime}(t)-(4+p)\left\|u_{t}\right\|^{2} \geq & -(4+2 p) E(0)+(p-(1+p)(1-l))\|\Delta u\|^{2} \\
& +(1+p)(g \odot \Delta u)(t),
\end{aligned}
$$

if we set $m:=(1+p)-\frac{1}{l}$ then inequality (4.8) yields the desired result.

Consequently, we have the following result.

Lemma 7 Assume that (A4) and (A5) hold. $u$ be a local solution of (1.1) and that either one of the following four conditions is satisfied:

(i) $E(0)<0$

(ii) $E(0)=0$ and $\psi^{\prime}(0)>0$

(iii) $0<E(0)<\frac{m}{p} E_{1 \text { and }} l^{\frac{1}{2}}\left\|\Delta u_{0}\right\|>\lambda_{1}$

(iv) $\frac{m}{p} E_{1} \leq E(0)$ and $\psi^{\prime}(0)>r_{2}\left[\psi(0)+\frac{(4+2 p) E(0)}{4+p}\right]$.

Then $\psi^{\prime}(t)>0$ for $t>t^{*}$, where

in case (i)

$$
t^{*}=\max \left\{0, \frac{\psi^{\prime}(0)}{(4+2 p) E(0)}\right\}
$$

in cases (ii), (iv)

$$
t^{*}=0,
$$

and in case (iii)

$$
t^{*}=\max \left\{0, \frac{-\psi^{\prime}(0)}{(4+2 p)\left(\frac{m}{p} E_{1}-E(0)\right)}\right\} .
$$


Proof Suppose that condition $(i)$ is satisfied. Then from (4.5), we have

$$
\psi^{\prime}(t) \geq \psi^{\prime}(0)-(4+2 p) E(0) t, \quad t \geq 0 .
$$

Thus $\psi^{\prime}(\mathrm{t})>0$ for $t>t^{*}$, and it is easy to see that $t^{*}$ satisfies (4.9).

If $E(0)=0$, then by using (4.3) we have $\psi^{\prime \prime}(t) \geq 0$, and since $\psi^{\prime}(0)>0$ we arrive at

$$
\psi^{\prime}(t)>0, \text { for } t>0 \text {. }
$$

If $0<E(0)<\frac{m}{p} E_{1}$ and $l^{\frac{1}{2}}\left\|\Delta u_{0}\right\|>\lambda_{1}$ then by Lemma 4 , we see that

$$
\begin{aligned}
m\left(l\|\Delta u\|^{2}+(g \odot \Delta u)(t)\right)-(4 & +2 p) E(0) \geq m \lambda_{2}^{2}-(4+2 p) E(0) \\
& >m \frac{4+2 p}{p} E_{1}-(4+2 p) E(0) \\
= & (4+2 p)\left[\frac{m}{p} E_{1}-E(0)\right] .
\end{aligned}
$$

Thus from (4.5), we have

$$
\begin{aligned}
\psi^{\prime \prime}(t) \geq & m\left(l\|\Delta u\|^{2}+(g \odot \Delta u)(t)\right)-(4+2 p) E(0) \\
> & (4+2 p)\left[\frac{m}{p} E_{1}-E(0)\right]>0,
\end{aligned}
$$

and integrating (4.12) from 0 to $t$ gives

$$
\psi^{\prime}(t)>0, \text { for } t \geq t^{*},
$$

where $t^{*}$ satisfies (4.11).

Let $\frac{m}{p} E_{1} \leq E(0)$, this assumption causes that

$$
\psi^{\prime \prime}(t)-(4+p)\left\|u_{t}\right\|^{2}+(4+2 p) E(0) \geq 0,
$$

and by using Hölder and Young's inequalities, we get

$$
\left\|u_{t}\right\|^{2} \geq \psi^{\prime}(t)-\psi(t)
$$

thus

$$
\psi^{\prime \prime}(t)-(4+p) \psi^{\prime}(t)+(4+p) \psi(t)+(4+2 p) E(0) \geq 0 .
$$

We see that the hypotheses of Lemma 2 are fulfilled with

$$
\delta=\frac{p}{4} \quad \text { and } \quad B(t)=\psi(t)+\frac{(4+2 p) E(0)}{4+p}
$$

and the conclusion of Lemma 2.2 gives us

$$
\psi^{\prime}(t)>0, \text { for } t>0 .
$$

Therefore the proof is complete.

To estimate the life-span of $\psi(t)$, we define the following functional

$$
Y(t)=\psi(t)^{-\frac{p}{4}}, \quad \text { for } \quad t \geq 0 .
$$


Then we have

$$
\begin{aligned}
& Y^{\prime}(t)=\frac{p}{4} Y(t)^{1+\frac{4}{p}} \psi^{\prime}(t), \\
& Y^{\prime \prime}(t)=-\frac{p}{4} Y(t)^{1+\frac{8}{p}}\left[\psi^{\prime \prime}(t) \psi(t)-\left(1+\frac{p}{4}\right)\left(\psi^{\prime}(t)\right)^{2}\right] .
\end{aligned}
$$

Using (4.4)-(4.6) and exploiting Holder's inequality on $\psi^{\prime}(t)$, we get

$$
\begin{aligned}
\psi^{\prime \prime} & (t) \psi(t)-\left(1+\frac{p}{4}\right)\left(\psi^{\prime}(t)\right)^{2} \\
\geq & {\left[\left(l\|\Delta u\|^{2}+(g \odot \Delta u)(t)\right)-(4+2 p) E(0)+(4+p)\left\|u_{t}\right\|^{2}\right] \psi(t) } \\
& -4\left(1+\frac{p}{4}\right)\left\|u_{t}\right\|^{2} \psi(t) \\
= & {\left[\left(l\|\Delta u\|^{2}+(g \odot \Delta u)(t)\right)-(4+2 p) E(0)\right] Y(t)^{\frac{-4}{p}} . }
\end{aligned}
$$

Utilizing the last inequality into (4.16) yields

$$
Y^{\prime \prime}(t) \leq-\frac{p}{4}\left[\left(l\|\Delta u\|^{2}+(g \odot \Delta u)(t)\right)-(4+2 p) E(0)\right] Y(t)^{1+\frac{4}{p}} .
$$

Now we should assume different values for initial energy $E(0)$.

(1) At first if $E(0) \leq 0$ then from (4.17) we have

$$
Y^{\prime \prime}(t) \leq \frac{p}{4}(4+2 p) E(0) Y(t)^{1+\frac{4}{p}},
$$

on the other hand by Lemma $7, Y^{\prime}(t)<0$ for $t>t^{*}$. Multiplying (4.18) by $Y^{\prime}(t)$ and integrating from $t^{*}$ to $t$, we deduce that

$$
Y^{\prime}(t)^{2} \geq \alpha+\beta Y(t)^{2+\frac{4}{p}} \quad \text { for } \quad t \geq t^{*},
$$

where

$$
\alpha=\frac{p^{2}}{16} Y\left(t^{*}\right)^{2+\frac{8}{p}}\left[\psi^{\prime}\left(t^{*}\right)^{2}-8 E(0) Y\left(t^{*}\right)^{-\frac{4}{p}}\right]>0,
$$

and

$$
\beta=\frac{p^{2}}{2} E(0)
$$

Then the hypotheses of Lemma 3 are fulfilled with $\delta=\frac{p}{4}, t_{0}=t^{*}$ and using the conclusion of Lemma 3 , there exists a finite time $T^{*}$ such that $\lim _{t \rightarrow T^{*}} Y(t)=0$, i.e., in this case some solutions blow up in finite time $T^{*}$.

(2) If $0<E(0)<\frac{m}{p} E_{1}$, then from (4.17) and (4.12) we have

$$
Y^{\prime \prime}(t) \leq-\frac{p}{4}(4+2 p)\left[\frac{m}{p} E_{1}-E(0)\right] Y(t)^{1+\frac{4}{p}}
$$

Then using the same arguments as in (1), we get

$$
Y^{\prime}(t)^{2} \geq \alpha_{1}+\beta_{1} Y(t)^{2+\frac{4}{p}} \quad \text { for } \quad t \geq t^{*},
$$


where

$$
\alpha_{1}=\frac{p^{2}}{16} Y\left(t^{*}\right)^{2+\frac{8}{p}}\left(\psi^{\prime}\left(t^{*}\right)^{2}+8\left[\frac{m}{p} E_{1}-E(0)\right] Y\left(t^{*}\right)^{-\frac{4}{p}}\right)>0,
$$

and

$$
\beta_{1}=\frac{p^{2}}{2}\left[E(0)-\frac{m}{p} E_{1}\right] .
$$

Thus by Lemma 3, there exists a finite time $T^{*}$ such that

$$
\lim _{t \rightarrow T *-} \psi(t)=\infty .
$$

(3) $\frac{m}{p} E_{1} \leq E(0)$. In this case, it is easy to see that by using (4.19) and (4.20) into discussion in part (1), we obtain

$$
\alpha>0 \quad \text { if and only if } \quad E(0)<\frac{\psi^{\prime}\left(t^{*}\right)^{2}}{8 \psi\left(t^{*}\right)} .
$$

Hence, Lemma 3 yields the blow-up property in this case.

Therefore, we proved the following theorem.

Theorem 2 Assume that (A4) and (A5) hold. $u$ be a local solution of (1.1) and that either one of the following four conditions is satisfied:

(i) $E(0)<0$

(ii) $E(0)=0$ and $\psi^{\prime}(0)>0$

(iii) $0<E(0)<\frac{m}{p} E_{1 \text { and }} l^{\frac{1}{2}}\left\|\Delta u_{0}\right\|>\lambda_{1}$

(iv) $\frac{m}{p} E_{1} \leq E(0)$ and $\psi^{\prime}(0)>r_{2}\left[\psi(0)+\frac{(4+2 p) E(0)}{4+p}\right]$ holds.

Then the solution $u$ blows up at finite time $T^{*}$. Moreover, the upper bounds for $T^{*}$ can be estimated according to the sign of $E(0)$ :

in case (i)

$$
T^{*} \leq t^{*}-\frac{Y\left(t^{*}\right)}{Y^{\prime}\left(t^{*}\right)} .
$$

Furthermore, if $Y\left(t^{*}\right)<\min \left\{1, \sqrt{\frac{\alpha}{-\beta}}\right\}$, then

$$
T^{*} \leq t^{*}+\frac{1}{\sqrt{-\beta}} \ln \frac{\sqrt{\frac{\alpha}{-\beta}}}{\sqrt{\frac{\alpha}{-\beta}}-Y\left(t^{*}\right)}
$$

in cases (ii)

$$
T^{*} \leq t^{*}-\frac{Y\left(t^{*}\right)}{Y^{\prime}\left(t^{*}\right)} \text { or } T^{*} \leq t^{*}+\frac{Y\left(t^{*}\right)}{\sqrt{\alpha}}
$$


in case (iii)

$$
T^{*} \leq t^{*}-\frac{Y\left(t^{*}\right)}{Y^{\prime}\left(t^{*}\right)}
$$

Furthermore, if $Y\left(t^{*}\right)<\min \left\{1, \sqrt{\frac{\alpha}{-\beta}}\right\}$, then

$$
T^{*} \leq t^{*}+\frac{1}{\sqrt{-\beta_{1}}} \ln \frac{\sqrt{\frac{\alpha_{1}}{-\beta_{1}}}}{\sqrt{\frac{\alpha_{1}}{-\beta_{1}}}-Y\left(t^{*}\right)}
$$

and in case (iv)

$$
T^{*} \leq \frac{Y\left(t^{*}\right)}{\sqrt{\alpha}} \text { or } T^{*} \leq t^{*}+2^{\frac{3 p+4}{2 p}} \frac{p c}{4 \sqrt{\alpha}}\left[1-\left(1+c Y\left(t^{*}\right)\right)^{\frac{-2}{p}}\right]
$$

where $d=\left(\frac{\beta}{\alpha}\right)^{\frac{p}{p+8}}$. Here $\alpha, \beta, \alpha_{1}$, and $\beta_{1}$ are given in (4.19)-(4.22), respectively. Note that each $t^{*}$ in the above cases satisfy the same case in Lemma 7.

\section{Acknowledgements}

The authors would like to thank the referees for the careful reading of this article and for the valuable suggestions to improve the presentation and style of the article. This study was supported by Shiraz University.

\section{Authors' contributions}

The authors declare that the study was realized in collaboration with the same responsibility. All authors read and approved the final manuscript.

\section{Competing interests}

The authors declare that they have no competing interests.

Received: 3 December 2011 Accepted: 26 April 2012 Published: 26 April 2012

\section{References}

1. Bayrak, V, Can, M, Aliyev, FA: Nonexistence of global solutions of a quasilinear hyperbolic equations. Math Inequal Appl. 1, 367-374 (1998)

2. Kalantarov, VK, Ladyzhenskaya, OA: Formation of collapses in quasilinear equations of parabolic and hyperbolic types. zap Nauchn Semin LOMI. 61, 77-102 (1977)

3. Messaoudi, SA: Blow-up of positive-initial-energy solutions of a nonlinear viscoelastic hyperbolic equation. J Math Anal Appl. 320, 902-915 (2006). doi:10.1016/j.jmaa.2005.07.022

4. Wu, ST: Energy decay rates via convexity for some second-order evolution equation with memory and nonlinear timedependent dissipation. Nonlinear Anal. 74, 532-543 (2011). doi:10.1016/..na.2010.09.007

5. Munoz Rivera, JE, Lapa, EC, Baretto, R: Decay rates for viscoelastic plates with memory. J Elasticity. 44, 61-87 (1996). doi:10.1007/BF00042192

6. Amroun, NE, Benaissa, A: Global existence and energy decay of solutions to a Petrovsky equation with general nonlinear dissipation and source term. Georg Math J. 13, 397-410 (2006)

7. Andrade, D, Fatori, LH, Rivera, JM: Nonlinear transmission problem with a dissipative boundary condition of memory type. Electron J Diff Equ. 53, 1-16 (2006)

8. Berrimi, S, Messaoudi, SA: Existence and decay of solutions of a viscoelastic equation with a nonlinear source. Nonlinear Anal. 64, 2314-2331 (2006). doi:10.1016/j.na.2005.08.015

9. Cavalcanti, MM, Domingos Cavalcanti, VN, Ma, TF, Soriano, JA: Global existence and asymptotic stability for viscoelastic problems. Diff Integ Equ. 15, 731-748 (2002)

10. Chen, CS, Ren, L: Weak solution for a fourth order nonlinear wave equation. J Southeast Univ (English Ed). 21, 369-374 (2005)

11. Li, MR, Tsai, LY: Existence and nonexistence of global solutions of some systems of semilinear wave equations. Nonlinear Anal. 54, 1397-1415 (2003). doi:10.1016/S0362-546X(03)00192-5

12. Messaoudi, SA: Global existence and nonexistence in a system of Petrovsky. J Math Anal Appl. 265, 296-308 (2002) doi:10.1006/jmaa.2001.7697 
13. Messaoudi, SA, Tatar, N: Exponential and polynomial decay for a quasilinear viscoelastic equation. Nonlinear Anal. 68, 785-793 (2008). doi:10.1016/j.na.2006.11.036

14. Wang, Y: A global nonexistence theorem for viscoelastic equations with arbitrary positive initial energy. Appl Math Lett. 22, 1394-1400 (2009). doi:10.1016/j.aml.2009.01.052

15. Han, X, Wang, M: Global existence and uniform decay for a nonlinear viscoelastic equation with damping. Nonlinear Anal. 70, 3090-3098 (2009). doi:10.1016/j.na.2008.04.011

16. Messaoudi, SA, Tatar, N: Global existence and uniform decay of solutions for a quasilinear viscoelastic problem. Math Methods Appl Sci. 30, 665-680 (2007). doi:10.1002/mma.804

17. Li, G, Sun, Y, Liu, W: Global existence, uniform decay and blow-up of solutions for a system of Petrovsky equations. Nonlinear Anal. 74, 1523-1538 (2011). doi:10.1016/j.na.2010.10.025

18. Adams, RA, Fournier, JJF: Sobolev Spaces, of Pure and Applied Mathematics (Amsterdam). Elsevier/Academic Press, Amsterdam, 2140 (2003)

19. Wu, ST, Tsai, LY: On global solutions and blow-up of solutions for a nonlinearly damped Petrovsky system. Taiwanese J Math. 13, 545-558 (2009)

doi:10.1186/1687-2770-2012-50

Cite this article as: Tahamtani and Shahrouzi: Existence and blow up of solutions to a Petrovsky equation with memory and nonlinear source term. Boundary Value Problems 2012 2012:50.

\section{Submit your manuscript to a SpringerOpen ${ }^{\circ}$} journal and benefit from:

- Convenient online submission

- Rigorous peer review

- Immediate publication on acceptance

- Open access: articles freely available online

- High visibility within the field

- Retaining the copyright to your article

Submit your next manuscript at $\gg$ springeropen.com 